\title{
Sigma decomposition: the CP-odd Lagrangian
}

\author{
I.M. Hierro, ${ }^{a}$ L. Merlo ${ }^{b}$ and S. Rigolin ${ }^{a}$ \\ ${ }^{a}$ Dipartimento di Fisica "G. Galilei", Università di Padova and INFN, Sezione di Padova, \\ Via Marzolo 8, I-35131 Padua, Italy \\ ${ }^{b}$ Instituto de Física Teórica, IFT-UAM/CSIC, Universidad Autónoma de Madrid, \\ Cantoblanco, 28049, Madrid, Spain \\ E-mail: ignacio.hierro@pd.infn.it, luca.merlo@uam.es, \\ stefano.rigolin@pd.infn.it
}

ABSTRaCt: In Alonso et al., JHEP 12 (2014) 034, the CP-even sector of the effective chiral Lagrangian for a generic composite Higgs model with a symmetric coset has been constructed, up to four momenta. In this paper, the CP-odd couplings are studied within the same context. If only the Standard Model bosonic sources of custodial symmetry breaking are considered, then at most six independent operators form a basis. One of them is the weak- $\theta$ term linked to non-perturbative sources of CP violation, while the others describe CP-odd perturbative couplings between the Standard Model gauge bosons and an Higgs-like scalar belonging to the Goldstone boson sector. The procedure is then applied to three distinct exemplifying frameworks: the original $\mathrm{SU}(5) / \mathrm{SO}(5)$ Georgi-Kaplan model, the minimal custodial-preserving $\mathrm{SO}(5) / \mathrm{SO}(4)$ model and the minimal $\mathrm{SU}(3) /(\mathrm{SU}(2) \times$ $\mathrm{U}(1)$ ) model, which intrinsically breaks custodial symmetry. Moreover, the projection of the high-energy electroweak effective theory to the low-energy chiral effective Lagrangian for a dynamical Higgs is performed, uncovering strong relations between the operator coefficients and pinpointing the differences with the elementary Higgs scenario.

Keywords: Beyond Standard Model, Higgs Physics, Technicolor and Composite Models

ArXiv EPrint: 1510.07899 


\section{Contents}

1 Introduction 1

2 CP effective chiral Lagrangian at the EW scale 3

$3 \quad$ CP effective chiral Lagrangian for symmetric cosets 5

$\begin{array}{lll}3.1 & \text { Basis of independent operators } & 7\end{array}$

3.2 General EW effective Lagrangian for a symmetric $\mathcal{G} / \mathcal{H}$ coset 8

4 Specific composite Higgs models $\quad 9$

4.1 The SU(5)/SO(5) composite Higgs model 9

4.1.1 The low-energy effective EW chiral Lagrangian 10

4.2 The minimal $\mathrm{SO}(5) / \mathrm{SO}(4)$ composite Higgs model 11

4.2.1 The low-energy effective EW chiral Lagrangian 12

4.3 The $\mathrm{SU}(3) /(\mathrm{SU}(2) \times \mathrm{U}(1))$ composite Higgs model 12

4.3.1 The low-energy effective EW chiral Lagrangian 13

5 Matching the high- and the low-energy Lagrangians $\quad 13$

$\begin{array}{lll}5.1 & \text { The small } \xi \text { limit } & 14\end{array}$

6 Conclusions 16

\section{Introduction}

A plausible solution to the electroweak (EW) hierarchy problem is provided in the socalled composite Higgs $(\mathrm{CH})$ scenario [1-6], where the Higgs particle arises as a pseudo Nambu-Goldstone Boson (GB) of a global symmetry breaking present at high energy. In this setup, the global symmetry group, denoted here $\mathcal{G}$, is spontaneously broken by some strong dynamics mechanism to a subgroup, $\mathcal{H}$, at a certain scale $\Lambda_{s}$ that for definiteness we assume around the TeV. Among the GBs arising from the breaking, three have to be identified with the would-be-longitudinal components of the Standard Model (SM) gauge bosons and one can be associated to a scalar field, playing the role of the Higgs field, $\varphi$. The characteristic scale of this global symmetry breaking, dubbed $f$, is related with the strong scale $\Lambda_{s}$ by the relation $\Lambda_{s} \leq 4 \pi f$ [7]. Due to the GBs shift symmetry, the Higgs develops a mass only at one-loop level due to an induced Coleman-Weinberg scalar potential: an elegant solution to the SM hierarchy problem is thus provided (for recent reviews see for example refs. $[8,9])$.

Smoking guns of specific CH models [10-13] are exotic resonances with masses smaller than $1.5 \mathrm{TeV}$ [14-21], that however have not been discovered at colliders yet. On the other hand, indirect studies on deviations from the SM predictions are viable strategies to test 
the presence of New Physics (NP). The most suitable tool in this case is the effective field theory approach, that model-independently provides a description of non-SM signals that could be seen at colliders.

When considering only the bosonic sector, the effective Lagrangian at the EW scale $v$ (defined by the $W$ mass $m_{W}=g v / 2$ ) can be written in terms of the SM gauge bosons the longitudinal components, represented by the GB matrix

$$
\mathbf{U}(x)=e^{i \sigma_{a} \pi^{a}(x) / v}
$$

and the transverse ones $W_{\mu \nu}$ and $B_{\mu \nu}$ - and of an isosinglet CP-even scalar $h$ representing the resonance discovered at LHC $[22,23]$. In the most general case, the couplings with $h$ can be described by a generic smooth function $\mathcal{F}(h)$ [24]. The complete effective basis of pure-gauge and gauge-Higgs interactions ${ }^{1}$ has been presented, respectively for the CP-even and CP-odd case, in refs. [24, 29], based on the Appelquist-Longhitano-Feruglio (ALF) basis [30-34], and following the spirit of refs. [35-37]. This low-energy effective Lagrangian represents a fundamental tool for Higgs studies at collider as shown in refs. [29, 38, 39], where the main focus was in disentangling an elementary Higgs from a composite one, by the analysis of its couplings. In the former case, the SM GBs are described together with the physical Higgs by the electroweak Higgs doublet, and this leads to correlations between certain observables and to the suppressions of the so-called anomalous couplings. On the other hand, in the composite case, the adimensionality of the GBs matrix $\mathbf{U}(x)$, and the treatment of $\mathbf{U}(x)$ and the Higgs field $h$ as independent objects translate into an additional decorrelations of observables and into the appearance of unsuppressed anomalous couplings.

The low-energy effective chiral Lagrangian described in refs. [24, 29] can be useful to describe an extended class of BSM "Higgs" models, from more extreme technicolor-like ansatzs to intermediate situations such as $\mathrm{CH}$ models or dilaton-like scalar frameworks. The distinct limits can be reached for different values of the GB scale $f$ and by fixing the value of the coefficient and choosing the specific $\mathcal{F}(h)$ function associated to each effective operator. On the other hand, it is often interesting to acquire a top-bottom perspective and connect the low-energy effective chiral Lagrangian with specific classes of $\mathrm{CH}$ models. This has been worked out in detail in ref. [40], where the CP conserving high-energy effective chiral Lagrangian for a generic symmetric coset $\mathcal{G} / \mathcal{H}$ has been constructed, up to four momenta. Three representative examples have been then analysed: the original $\mathrm{SU}(5) / \mathrm{SO}(5)$ Georgi-Kaplan model, the minimal custodial-preserving $\mathrm{SO}(5) / \mathrm{SO}(4)$ model and the minimal $\mathrm{SU}(3) /(\mathrm{SU}(2) \times \mathrm{U}(1))$ model, which intrinsically breaks custodial symmetry. The projection at low-energy of the effective Lagrangian for each of the aforementioned $\mathrm{CH}$ models is shown to match the chiral effective Lagrangian for a dynamical Higgs of refs. [24], uncovering strong relations between the operator coefficients.

The aim of this paper is to complete the study performed in ref. [40] by introducing the CP-odd effective chiral Lagrangian, up to four derivatives for a generic symmetric coset. The analysis is then detailed for the same three representative $\mathrm{CH}$ models as in ref. [40]. This completes the tool necessary to study exotic gauge-Higgs couplings at colliders and at

\footnotetext{
${ }^{1}$ Fermionic operators have been discussed at different levels and with different aims in refs. [25-28].
} 
low-energy experiments, and to investigate the nature of the Higgs particle and the origin of the EWSB mechanism.

The paper is organised as follows. Section 2 is devoted to recalling the low-energy effective chiral Lagrangian introduced in refs. [24, 29], focusing on the CP-odd couplings. Section 3 contains the high-energy effective chiral Lagrangian, describing the CP-odd interactions among SM gauge bosons and the GBs associated to the symmetric coset $\mathcal{G} / \mathcal{H}$. Only operators with at most four derivatives are retained in the Lagrangian. Furthermore, no source of custodial breaking besides the (gauge) SM one is considered. In sections 4.1, 4.2 and 4.3, the low-energy effective EW chiral Lagrangian is then derived from the high-energy one for the $\mathrm{SU}(5) / \mathrm{SO}(5), \mathrm{SO}(5) / \mathrm{SO}(4)$ and $\mathrm{SU}(3) /(\mathrm{SU}(2) \times \mathrm{U}(1))$ composite Higgs models. Finally in section 5, the connection with the low-energy chiral Lagrangian describing the linearly realised EW symmetry scenario (dubbed "linear Lagrangian" from now on), written in terms of the SM SU $(2)_{L}$-doublet Higgs, is also discussed. Conclusions are presented in section 6 .

\section{CP effective chiral Lagrangian at the EW scale}

The Higgsless EW effective chiral Lagrangian described in refs. [30-34] can be written in terms of the SM gauge bosons $W_{\mu}^{a}(x)$ and $B_{\mu}(x)$, the SM GBs matrix $\mathbf{U}(x)$ and its covariant derivative:

$$
\mathbf{D}_{\mu} \mathbf{U}(x) \equiv \partial_{\mu} \mathbf{U}(x)+i g \mathbf{W}_{\mu}(x) \mathbf{U}(x)-\frac{i g^{\prime}}{2} B_{\mu}(x) \mathbf{U}(x) \sigma_{3},
$$

where $\mathbf{W}_{\mu}(x) \equiv W_{\mu}^{a}(x) \sigma_{a} / 2$. To write the effective chiral operators it is convenient to introduce the following (pseudo-)scalar and vector chiral fields:

$$
\mathbf{T}(x) \equiv \mathbf{U}(x) \sigma_{3} \mathbf{U}^{\dagger}(x), \quad \mathbf{V}_{\mu}(x) \equiv\left(\mathbf{D}_{\mu} \mathbf{U}(x)\right) \mathbf{U}^{\dagger}(x) .
$$

Recalling the transformation property of $\mathbf{U}(x)$ under a (global) $\mathrm{SU}(2)_{L, R}$ transformation:

$$
\mathbf{U}(x) \rightarrow L \mathbf{U}(x) R^{\dagger}
$$

it follows that both $\mathbf{T}(x)$ and $\mathbf{V}(x)$ transform in the adjoint of $\mathrm{SU}(2)_{L}$,

$$
\mathbf{T}(x) \rightarrow L \mathbf{T}(x) L^{\dagger}, \quad \mathbf{V}_{\mu}(x) \rightarrow L \mathbf{V}_{\mu}(x) L^{\dagger} .
$$

These chiral fields (and their derivatives) together with the EW gauge bosons are the necessary building blocks to construct the (Higgsless) ALF basis.

After the discovery of the new light scalar degree of freedom (aka Higgs particle) one is forced to extend the previous basis, including all possible couplings between the Higgs, taken in all generalities as a CP-even scalar singlet field $h$, the SM gauge bosons and the (pseudo-)scalar and vector chiral fields. The effective chiral Lagrangian at the EW scale, describing the gauge and gauge-Higgs interactions, up to four derivatives, has been derived in ref. [24, 29], for the CP-even and CP-odd sectors respectively. In the following, we will focus only on the CP-odd sector, and we list here the full set of operators [29] necessary to describe the CP-odd bosonic interactions, organising them by their number of derivatives and their custodial character: 
CP-odd operators with two derivatives

Custodial preserving

Custodial breaking

$$
\mathcal{S}_{2 D}=i \frac{v^{2}}{4} \operatorname{Tr}\left(\mathbf{T} \mathcal{D}_{\mu} \mathbf{V}^{\mu}\right)
$$

\section{CP-odd operators with four derivatives}

$$
\begin{array}{ll}
\text { Custodial preserving } & \text { Custodial breaking } \\
\hline \mathcal{S}_{B B^{*}}=-\frac{g^{\prime 2}}{4} B_{\mu \nu}^{*} B^{\mu \nu} & \mathcal{S}_{4}=g \operatorname{Tr}\left(\mathbf{W}^{\mu \nu} \mathbf{V}_{\mu}\right) \operatorname{Tr}\left(\mathbf{T} \mathbf{V}_{\nu}\right) \\
\mathcal{S}_{W W^{*}}=-\frac{g^{2}}{2} \operatorname{Tr}\left(\mathbf{W}_{\mu \nu}^{*} \mathbf{W}^{\mu \nu}\right) & \mathcal{S}_{5}=i \operatorname{Tr}\left(\mathbf{V}^{\mu} \mathbf{V}^{\nu}\right) \operatorname{Tr}\left(\mathbf{T} \mathbf{V}_{\mu}\right) \partial_{\nu}(h / v) \\
& \mathcal{S}_{6}=i \operatorname{Tr}\left(\mathbf{V}^{\mu} \mathbf{V}_{\mu}\right) \operatorname{Tr}\left(\mathbf{T} \mathbf{V}^{\nu}\right) \partial_{\nu}(h / v) \\
\mathcal{S}_{1}=2 g g^{\prime} B_{\mu \nu}^{*} \operatorname{Tr}\left(\mathbf{T} \mathbf{W}^{\mu \nu}\right) & \mathcal{S}_{7}=g \operatorname{Tr}\left(\mathbf{T}\left[\mathbf{W}^{\mu \nu}, \mathbf{V}_{\mu}\right]\right) \partial_{\nu}(h / v) \\
\mathcal{S}_{2}=2 i g^{\prime} B_{\mu \nu}^{*} \operatorname{Tr}\left(\mathbf{T} \mathbf{V}^{\mu}\right) \partial^{\nu}(h / v) & \mathcal{S}_{8}=2 g^{2} \operatorname{Tr}\left(\mathbf{T} \mathbf{W}_{\mu \nu}^{*}\right) \operatorname{Tr}\left(\mathbf{T} \mathbf{W}_{\mu \nu}\right) \\
\mathcal{S}_{3}=2 i g \operatorname{Tr}\left(\mathbf{W}_{\mu \nu}^{*} \mathbf{V}_{\mu}\right) \partial_{\nu}(h / v) & \mathcal{S}_{9}=2 i g \operatorname{Tr}\left(\mathbf{W}_{\mu \nu}^{*} \mathbf{T}\right) \operatorname{Tr}\left(\mathbf{T} \mathbf{V}_{\mu}\right) \partial_{\nu}(h / v) \\
& \mathcal{S}_{10}=i \operatorname{Tr}\left(\mathbf{V}^{\mu} \mathcal{D}^{\nu} \mathbf{V}_{\nu}\right) \operatorname{Tr}\left(\mathbf{T} \mathbf{V}_{\mu}\right) \\
& \mathcal{S}_{11}=i \operatorname{Tr}\left(\mathbf{T} \mathcal{D}^{\mu} \mathbf{V}_{\mu}\right) \operatorname{Tr}\left(\mathbf{V}^{\nu} \mathbf{V}_{\nu}\right) \\
& \mathcal{S}_{12}=i \operatorname{Tr}\left(\left[\mathbf{V}^{\mu}, \mathbf{T}\right] \mathcal{D}^{\nu} \mathbf{V}_{\nu}\right) \partial_{\mu}(h / v) \\
& \mathcal{S}_{13}=i \operatorname{Tr}\left(\mathbf{T} \mathcal{D}^{\mu} \mathbf{V}_{\mu}\right) \partial^{\nu} \partial_{\nu}(h / v) \\
& \mathcal{S}_{14}=i \operatorname{Tr}\left(\mathbf{T} \mathcal{D}^{\mu} \mathbf{V}_{\mu}\right) \partial^{\nu}(h / v) \partial_{\nu}(h / v) \\
& \mathcal{S}_{15}=i \operatorname{Tr}\left(\mathbf{T} \mathbf{V}^{\mu}\right)\left(\operatorname{Tr}\left(\mathbf{T} \mathbf{V}^{\nu}\right)\right)^{2} \partial_{\mu}(h / v) \\
& \mathcal{S}_{16}=i \operatorname{Tr}\left(\mathbf{T} \mathcal{D}^{\mu} \mathbf{V}_{\mu}\right)\left(\operatorname{Tr}\left(\mathbf{T} \mathbf{V}^{\nu}\right)\right)^{2}
\end{array}
$$

where $X_{\mu \nu}^{*} \equiv \epsilon_{\mu \nu \rho \sigma} X^{\rho \sigma}$ for $X \equiv\{B, \mathbf{W}\}$ and $\mathcal{D}_{\mu}$ denotes the covariant derivative in the adjoint representation of $\mathrm{SU}(2)_{L}$, i.e.

$$
\mathcal{D}_{\mu} \mathbf{V}_{\nu} \equiv \partial_{\mu} \mathbf{V}_{\nu}+i g\left[\mathbf{W}_{\mu}, \mathbf{V}_{\nu}\right]
$$

The operators on the left column are custodial preserving, meaning that they do not introduce custodial breaking contributions distinct from the SM ones; instead, the operators on the right column encode tree-level custodial breaking sources beyond the SM (gauge) one. A common aspect of the operator in the last class is the presence of the scalar chiral field $\mathbf{T}(x)$ not in association with the $B_{\mu \nu}$ field strength. Notice, however, that the presence of $\mathbf{T}(x)$ inside an operator does automatically implies that it violates the custodial symmetry. For example, it is straightforward to verify that

$$
\epsilon_{\mu \nu \rho \sigma} \operatorname{Tr}\left(\mathbf{T} \mathbf{V}^{\mu} \mathbf{V}^{\nu}\right) \operatorname{Tr}\left(\mathbf{T} \mathbf{V}^{\rho}\right) \partial^{\sigma} h=\frac{2}{3} \epsilon_{\mu \nu \rho \sigma} \operatorname{Tr}\left(\mathbf{V}^{\mu} \mathbf{V}^{\nu} \mathbf{V}^{\rho}\right) \partial^{\sigma} h
$$

which shows that the presence of $\mathbf{T}(x)$ on the l.h.s. does not imply a custodial breaking nature of the operator, as it can be rewritten as an obviously custodial preserving one. 
This happens only for the operator on the l.h.s. of eq. (2.8), due to the presence of two $\mathbf{T}(x)$ in that specific combination.

The CP-odd (Higgless) ALF basis can be obtained from eqs. (2.5) and (2.6), disregarding all the operators containing one or more derivatives of $h$. Specifically the ALF CP-odd basis consists of one operator with two derivatives, $\mathcal{S}_{2 D}$, plus one custodial preserving and five custodial breaking operators with four-derivatives, besides the topological ones.

The low-energy electroweak chiral Lagrangian describing the CP-odd gauge, gaugeGoldstone and the gauge-Higgs interactions can instead be written as:

$$
\mathscr{L}_{\text {low }, \mathscr{}, \mathscr{P}}=\mathscr{L}_{\text {low }, \mathscr{p}}^{p^{2}}+\mathscr{L}_{\text {low }, \mathscr{p}}^{p^{4}},
$$

where $\mathscr{L}_{\text {low,CP }}^{p^{2}}$ and $\mathscr{L}_{\text {low,CP }}^{p^{4}}$ contain two and four-derivative operators, respectively,

$$
\begin{aligned}
& \mathscr{L}_{\text {low, }, \mathscr{p}}^{p^{2}}=c_{2 D} \mathcal{S}_{2 D} \mathcal{F}_{2 D}(h), \\
& \mathscr{L}_{\text {low, }, \mathscr{p}}^{p^{4}}=\mathcal{S}_{B B^{*}} \mathcal{F}_{B B^{*}}(h)+\mathcal{S}_{W W^{*}} \mathcal{F}_{W W^{*}}(h)+\sum_{i=1}^{16} c_{i} \mathcal{S}_{i} \mathcal{F}_{i}(h),
\end{aligned}
$$

with the functions $\mathcal{F}_{i}(h)$ encoding a generic dependence on $h .^{2}$ The careful reader should be warned about the slightly different notation used here with respect to that used in refs. [24, 29] for the definition of the $\mathcal{F}_{i}(h)$ functions. Here, the operators $\mathcal{P}_{i}$ do not contain the $\mathcal{F}_{i}(h)$ functions, which are instead left outside as multiplicative terms, and the only dependence on $h$ kept inside the operators $\mathcal{P}_{i}$ is $\partial h$. Furthermore, in refs. [24, 29], it was made explicit the dependence on a parameter that qualifies the degree of non-linearity of the theory: this parameter is usually labelled $\xi$ and defined as

$$
\xi \equiv(v / f)^{2}
$$

This parameter is meaningful only when the high-energy theory that leads to the lowenergy effective chiral Lagrangian is specified. Here, to keep the discussion as general as possible, the $\xi$ weights are reabsorbed in the coefficients $c_{i}$ and in the functions $\mathcal{F}_{i}(h)$. The role of $\xi$ will become clear in the following sections, once specific dynamical Higgs models will be considered.

\section{CP effective chiral Lagrangian for symmetric cosets}

In this section, the high-energy CP-odd effective Lagrangian will be constructed for a generic CH setup. The discussion will closely follow ref. [40], to which the reader is referred to for further details. Only few definitions will be recalled here and the notation will be fixed.

Consider a generic $\mathrm{CH}$ framework and denote with $\mathcal{G}$ the global symmetry group, spontaneously broken by some strong dynamics mechanism at the scale $\Lambda_{s}$, to a subgroup $\mathcal{H}$. The coset $\mathcal{G} / \mathcal{H}$ is assumed to be symmetric (see [41]): this does not restrict the

\footnotetext{
${ }^{2}$ Be aware that no derivatives of $h$ are included in $\mathcal{F}_{i}(h)$.
} 
applicability of the results of this analysis as all the known existing $\mathrm{CH}$ models satisfy this condition. The minimum requisite on the choice of specific $\mathcal{G}$ and $\mathcal{H}$ is that $\operatorname{dim}(\mathcal{G} / \mathcal{H}) \geq 4$, i.e. at least four GBs arise from the global symmetry breaking, such that three of them would be then identified with the longitudinal components of the SM gauge bosons and one with the light scalar resonance observed at LHC. No fermionic operators will be considered here, and only CP-odd ones will be retained among the set of bosonic operators, up to four derivatives. Moreover, we assume that the only sources of custodial breaking are the ones of the SM.

The GBs arising from the global spontaneous symmetry breaking $\mathcal{G} \rightarrow \mathcal{H}$ can be described by a field $\Omega(x)$ defined as $[42,43]$

$$
\Omega(x) \equiv e^{i \Xi(x) / 2 f},
$$

transforming under the global groups $\mathcal{G}$ and $\mathcal{H}$ as

$$
\Omega(x) \rightarrow \mathfrak{g} \Omega(x) \mathfrak{h}^{-1}(\Xi, \mathfrak{g}) .
$$

This expression defines the non-linear transformation of $\Xi(x): \mathfrak{g}$ represents a (global) element of $\mathcal{G}$, while $\mathfrak{h}(\Xi, \mathfrak{g})$ a (local) element of $\mathcal{H}$, which depends explicitly on $\mathfrak{g}$ and on the Goldstone boson field $\Xi(x)$. Notice that, for sake of brevity, $\mathfrak{h}(\Xi, \mathfrak{g})$ will be simply denoted as $\mathfrak{h}$ in the following.

The GB field matrix $\Xi(x)$ can be explicitly written in terms of the generators of the $\operatorname{coset} \mathcal{G} / \mathcal{H}, X_{\hat{a}}($ with $\hat{a}=1, \ldots, \operatorname{dim}(\mathcal{G} / \mathcal{H}))$, as

$$
\Xi(x)=\Xi^{\hat{a}}(x) X_{\hat{a}}
$$

The $X_{\hat{a}}$ generators together with the generators of the preserved subgroup $\mathcal{H}, T_{a}$ (with $a=1, \ldots, \operatorname{dim}(\mathcal{H}))$, form an orthonormal basis of $\mathcal{G}$.

The peculiarity of symmetric cosets is to admit an automorphism (usually dubbed "grading") that acts on the generators of $\mathcal{G}$ as

$$
\mathcal{R}:\left\{\begin{array}{l}
T_{a} \rightarrow+T_{a} \\
X_{\hat{a}} \rightarrow-X_{\hat{a}}
\end{array}\right.
$$

In a generic symmetric coset it is then possible to define a "squared" non-linear field $\boldsymbol{\Sigma}(x)$ :

$$
\boldsymbol{\Sigma}(x) \equiv \Omega(x)^{2},
$$

transforming under $\mathcal{G}$ as,

$$
\Sigma(x) \rightarrow \mathfrak{g} \boldsymbol{\Sigma}(x) \mathfrak{g}_{\mathcal{R}}^{-1} .
$$

From eq. (3.4) one immediately recovers that the GB field matrices $\Omega(x)$ and $\boldsymbol{\Sigma}(x)$ transform, under the discrete grading $\mathcal{R}$ automorphism, respectively, as:

$$
\Omega(x) \rightarrow \Omega(x)^{-1}, \quad \boldsymbol{\Sigma}(x) \rightarrow \boldsymbol{\Sigma}(x)^{-1} .
$$


This shows the equivalence in using $\Omega(x)$ or $\boldsymbol{\Sigma}(x)$ in the description of GBs degrees of freedom and its interactions, in frameworks with a symmetric coset $\mathcal{G} / \mathcal{H}$. Here, we will follow ref. [40] and adopt the description of the CP-odd operators using the $\Sigma$-representation.

To introduce gauge interactions it is convenient to formally gauge the full group $\mathcal{G}$. In the symmetric coset case, it is possible to define both the $\mathcal{G}$ gauge fields $\widetilde{\mathbf{S}}_{\mu}$, and the graded siblings $\widetilde{\mathbf{S}}_{\mu}^{\mathcal{R}} \equiv \mathcal{R}\left(\widetilde{\mathbf{S}}_{\mu}\right)$, transforming under $\mathcal{G}$, respectively, as:

$$
\widetilde{\mathbf{S}}_{\mu} \rightarrow \mathfrak{g} \widetilde{\mathbf{S}}_{\mu} \mathfrak{g}^{-1}-\frac{i}{g_{S}} \mathfrak{g}\left(\partial_{\mu} \mathfrak{g}^{-1}\right), \quad \widetilde{\mathbf{S}}_{\mu}^{\mathcal{R}} \rightarrow \mathfrak{g}_{\mathcal{R}} \widetilde{\mathbf{S}}_{\mu}^{\mathcal{R}} \mathfrak{g}_{\mathcal{R}}^{-1}-\frac{i}{g_{S}} \mathfrak{g}_{\mathcal{R}}\left(\partial_{\mu} \mathfrak{g}_{\mathcal{R}}^{-1}\right)
$$

with $g_{S}$ denoting the associated gauge coupling constant. The covariant derivative of the non-linear field $\boldsymbol{\Sigma}(x)$ and the chiral vector field can then be defined as:

$$
\begin{aligned}
\mathbf{D}_{\mu} \boldsymbol{\Sigma} & =\partial_{\mu} \boldsymbol{\Sigma}+i g_{S}\left(\widetilde{\mathbf{S}}_{\mu} \boldsymbol{\Sigma}-\boldsymbol{\Sigma} \widetilde{\mathbf{S}}_{\mu}^{\mathcal{R}}\right) \\
\widetilde{\mathbf{V}}_{\mu} & =\left(\mathbf{D}_{\mu} \boldsymbol{\Sigma}\right) \boldsymbol{\Sigma}^{-1}
\end{aligned}
$$

The following three $\mathcal{G}$-covariant objects can thus be used as building blocks for the (gauged) effective chiral Lagrangian:

$$
\widetilde{\mathbf{V}}_{\mu}, \quad \widetilde{\mathbf{S}}_{\mu \nu} \text { and } \Sigma \widetilde{\mathbf{S}}_{\mu \nu}^{\mathcal{R}} \boldsymbol{\Sigma}^{-1}
$$

The introduction of the graded vector chiral field $\widetilde{\mathbf{V}}_{\mu}^{\mathcal{R}}$ does not add any further independent structure, as indeed

$$
\tilde{\mathbf{V}}_{\mu}^{\mathcal{R}} \equiv \mathcal{R}\left(\widetilde{\mathbf{V}}_{\mu}\right)=\left(\mathbf{D}_{\mu} \boldsymbol{\Sigma}\right)^{-1} \boldsymbol{\Sigma} \quad \text { with } \quad \boldsymbol{\Sigma} \widetilde{\mathbf{V}}_{\mu}^{\mathcal{R}} \boldsymbol{\Sigma}^{-1}=-\widetilde{\mathbf{V}}_{\mu}
$$

Under the hypothesis of absence of any custodial symmetry breaking source besides the SM ones, any operator containing the high-energy sibling of the scalar chiral field $\mathbf{T}(x)$,

$$
\widetilde{\mathbf{T}} \equiv \boldsymbol{\Sigma} Q_{Y} \boldsymbol{\Sigma}^{-1},
$$

with $Q_{Y}$ being the embedding in $\mathcal{G}$ of the hypercharge generator, should not enter in the basis, except in one specific case, discussed later on, where the presence of two $\widetilde{\mathbf{T}}$ gives rise to a custodial preserving operator.

\subsection{Basis of independent operators}

Performing an expansion in momenta and keeping operators with at most four derivatives, one can write the following independent structures:

\section{4-momenta CP-odd operators with gauge field strength $\widetilde{\mathbf{S}}_{\mu \nu}$}

$$
\operatorname{Tr}\left(\widetilde{\mathbf{S}}_{\mu \nu}^{*} \widetilde{\mathbf{S}}^{\mu \nu}\right), \quad \operatorname{Tr}\left(\widetilde{\mathbf{S}}_{\mu \nu}^{*} \boldsymbol{\Sigma} \widetilde{\mathbf{S}}^{\mu \nu, R} \boldsymbol{\Sigma}^{-1}\right),
$$

The first operator resembles the usual $\theta$ term operator for QCD. The other contains gauge-GB and pure-gauge interactions. 


\section{4-momenta CP-odd operators without gauge field strength $\widetilde{\mathbf{S}}_{\mu \nu}$}

$$
\epsilon_{\mu \nu \rho \sigma} \operatorname{Tr}\left(\widetilde{\mathbf{T}}\left[\widetilde{\mathbf{V}}^{\mu}, \widetilde{\mathbf{V}}^{\nu}\right]\right) \operatorname{Tr}\left(\widetilde{\mathbf{T}}\left[\widetilde{\mathbf{V}}^{\rho}, \tilde{\mathbf{V}}^{\sigma}\right]\right), \quad \epsilon_{\mu \nu \rho \sigma} \operatorname{Tr}\left(\tilde{\mathbf{V}}^{\mu} \tilde{\mathbf{V}}^{\nu} \tilde{\mathbf{V}}^{\rho} \tilde{\mathbf{V}}^{\sigma}\right),
$$

Other operators with traces of two $\tilde{\mathbf{V}}$ 's are clearly vanishing due to antisymmetry.

The operators listed in eqs. (3.14)-(3.15) represent the set of independent CP-odd structures that can be introduced in a generic symmetric coset. Other operators could be easily introduced, apparently giving rise to new independent structures, most notably:

$$
\operatorname{Tr}\left(\widetilde{\mathbf{S}}_{\mu \nu}^{*}\left[\widetilde{\mathbf{V}}^{\mu}, \widetilde{\mathbf{V}}^{\nu}\right]\right), \quad \epsilon_{\mu \nu \rho \sigma} \operatorname{Tr}\left(\left(\mathcal{D}^{\mu} \widetilde{\mathbf{V}}^{\nu}\right)\left(\mathcal{D}^{\rho} \widetilde{\mathbf{V}}^{\sigma}\right)\right), \quad \epsilon_{\mu \nu \rho \sigma} \operatorname{Tr}\left(\left(\mathcal{D}^{\mu} \widetilde{\mathbf{V}}^{\nu}\right) \tilde{\mathbf{V}}^{\rho} \widetilde{\mathbf{V}}^{\sigma}\right),
$$

where the adjoint covariant derivative acting on $\widetilde{\mathbf{V}}^{\mu}$ is defined as

$$
\mathcal{D}_{\mu} \widetilde{\mathbf{V}}_{\nu}=\partial_{\mu} \widetilde{\mathbf{V}}_{\nu}+i g_{S}\left[\widetilde{\mathbf{S}}_{\mu}, \widetilde{\mathbf{V}}_{\nu}\right] .
$$

However, making use of the Bianchi identity and of the well known properties of the commutator of the covariant derivatives, it is possible to show that all these operators do not introduce any independent structure besides the one listed in eqs. (3.14)-(3.15). It is also worth noticing that in specific $\mathcal{G} / \mathcal{H}$ realizations, some of the operators listed may not be independent. For example the operator with traces of four $\widetilde{\mathbf{V}}^{\mu}$ appearing on the right hand side of eq. (3.15) is redundant in all the considered $\mathrm{CH}$ models even if it was not the case for its CP-even counterpart. Moreover, other operators containing even powers of $\widetilde{\mathbf{T}}$ could be considered in the list above, but it is straightforward to show that they introduce custodial breaking sources beyond the SM ones, and therefore are not considered. ${ }^{3}$

\subsection{General EW effective Lagrangian for a symmetric $\mathcal{G} / \mathcal{H}$ coset}

The operators listed in eqs. (3.14)-(3.15) have been obtained under the hypothesis of gauging the full group $\mathcal{G}$. In most of the $\mathrm{CH}$ realisations, however, only the SM gauge group is gauged, while the group $\mathcal{G}$ is global. In these cases, the generic gauge field $\widetilde{\mathbf{S}}_{\mu}$ should contain only the components corresponding to the EW symmetry.

Specifying the dependence on the EW gauge bosons does not lead to new operator structures in the sector made out exclusively of $\widetilde{\mathbf{V}}_{\mu}$ fields (see eq. (3.15)), while all operators where the gauge field strength appears explicitly, such as those in eq. (3.14), should be "doubled" by substituting $\widetilde{\mathbf{S}}_{\mu}$ either with $\widetilde{\mathbf{W}}_{\mu}$ or $\widetilde{\mathbf{B}}_{\mu}$, defined by

$$
\widetilde{\mathbf{W}}_{\mu} \equiv W_{\mu}^{a} Q_{L}^{a} \quad \text { and } \quad \widetilde{\mathbf{B}}_{\mu} \equiv B_{\mu} Q_{Y}
$$

where $Q_{L}^{a}$ and $Q_{Y}$ denote the embedding in $\mathcal{G}$ of the $\mathrm{SU}(2)_{L} \times \mathrm{U}(1)_{Y}$ generators. It follows that a larger number of independent invariants can be written in this case. In consequence, the CP-odd EW high-energy chiral Lagrangian describing bosonic interactions, up to four derivatives, contains in total six operators:

$$
\mathscr{L}_{\text {high }, \varnothing}=\tilde{c}_{W W^{*}} \widetilde{\mathcal{B}}_{W W^{*}}+\tilde{c}_{B \Sigma^{*}} \widetilde{\mathcal{B}}_{B \Sigma^{*}}+\tilde{c}_{W \Sigma^{*}} \widetilde{\mathcal{B}}_{W \Sigma^{*}}+\tilde{c}_{1} \widetilde{\mathcal{B}}_{1}+\tilde{c}_{2} \widetilde{\mathcal{B}}_{2}+\tilde{c}_{3} \widetilde{\mathcal{B}}_{3},
$$

\footnotetext{
${ }^{3}$ We thank A. Wulzer for the discussions that led to include the operator on the right hand side of eq. (3.15) in the list of independent operators.
} 
with

$$
\begin{aligned}
& \widetilde{\mathcal{B}}_{W W^{*}}=-\frac{g^{2}}{4} \operatorname{Tr}\left(\widetilde{\mathbf{W}}_{\mu \nu}^{*} \widetilde{\mathbf{W}}^{\mu \nu}\right) \quad \widetilde{\mathcal{B}}_{1}=g g^{\prime} \operatorname{Tr}\left(\widetilde{\mathbf{W}}_{\mu \nu}^{*} \boldsymbol{\Sigma} \widetilde{\mathbf{B}}^{\mu \nu} \boldsymbol{\Sigma}^{-1}\right) \\
& \widetilde{\mathcal{B}}_{B \Sigma^{*}}=g^{\prime 2} \operatorname{Tr}\left(\widetilde{\mathbf{B}}_{\mu \nu}^{*} \boldsymbol{\Sigma} \widetilde{\mathbf{B}}^{\mu \nu} \boldsymbol{\Sigma}^{-1}\right) \quad \widetilde{\mathcal{B}}_{2}=\epsilon_{\mu \nu \rho \sigma} \operatorname{Tr}\left(\widetilde{\mathbf{T}}\left[\widetilde{\mathbf{V}}^{\mu}, \widetilde{\mathbf{V}}^{\nu}\right]\right) \operatorname{Tr}\left(\widetilde{\mathbf{T}}\left[\widetilde{\mathbf{V}}^{\rho}, \widetilde{\mathbf{V}}^{\sigma}\right]\right) \\
& \widetilde{\mathcal{B}}_{W \Sigma^{*}}=g^{2} \operatorname{Tr}\left(\widetilde{\mathbf{W}}_{\mu \nu}^{*} \boldsymbol{\Sigma} \widetilde{\mathbf{W}}^{\mu \nu} \boldsymbol{\Sigma}^{-1}\right) \quad \widetilde{\mathcal{B}}_{3}=\epsilon_{\mu \nu \rho \sigma} \operatorname{Tr}\left(\widetilde{\mathbf{V}}^{\mu} \widetilde{\mathbf{V}}^{\nu} \widetilde{\mathbf{V}}^{\rho} \widetilde{\mathbf{V}}^{\sigma}\right) .
\end{aligned}
$$

According to the effective field theory approach, all the coefficients $\tilde{c}_{i}$ are expected to be of the same order of magnitude.

Notice that the first operator listed in eq. (3.18), $\widetilde{\mathcal{B}}_{W W^{*}}$, is a topological structure, analogous to the QCD $\theta$-term. Even if this coupling is usually not considered in the SM context, it is included here in the basis for sake of completeness as it could give rise to non-vanishing effects when considering the incorporation of fermions. On the other side, a similar term for the $\mathrm{U}(1)_{Y}$ is identically vanishing.

Notwithstanding $\theta$-terms, $\mathscr{L}_{\text {high.,®P }}$ contains five independent operators, and thus at most five arbitrary coefficients $\tilde{c}_{i}$ need to be introduced. They will govern the projection of $\mathscr{L}_{\text {high, } \mathscr{C P}}$ into $\mathscr{L}_{\text {low } \mathscr{C} \text {, }}$, in addition to $\xi$. Of course these considerations hold only at tree-level. As the gauging of the SM symmetry breaks explicitly the custodial and the grading symmetries, custodial and/or grading symmetry breaking operators will arise once quantum corrections induced by SM interactions are going to be considered. But this is beyond the scope of this paper.

In the next sections, three exemplifying $\mathrm{CH}$ models will be considered.

\section{Specific composite Higgs models}

In this section, we "decompose" the high-energy CP-odd operators of $\mathscr{L}_{\text {high,\&® }}$ described in the previous section in terms of the low-energy operators of the EW effective chiral Lagrangian $\mathscr{L}_{\text {low }, \infty}$, recalled in section 2 . In particular we consider the following three representative $\mathrm{CH}$ models: the $\mathrm{SU}(5) / \mathrm{SO}(5)$ Georgi and Kaplan model [5], the $\mathrm{SO}(5) / \mathrm{SO}(4)$ Minimal (custodial preserving) Composite Higgs Model [11] and the Minimal (custodial breaking) $\mathrm{SU}(3) /(\mathrm{SU}(2) \times \mathrm{U}(1))$ one.

The following procedure consists in "decomposing" the high-energy GB field $\boldsymbol{\Sigma}(x)$ defined in eq. (3.5) in terms of its SM and BSM scalar components (if present), and then projecting into the SM d.o.f. (the three would be GBs and the Higgs particle), assuming that the extra d.o.f. can be safely decoupled from the observed low-energy spectrum.

\subsection{The SU(5)/SO(5) composite Higgs model}

The first $\mathrm{CH}$ model was proposed by Georgi and Kaplan [5] more than 30 years ago and encodes the spontaneous symmetry breaking $\mathrm{SU}(5) \rightarrow \mathrm{SO}(5)$. It is a non-minimal model in terms of d.o.f. as fourteen GBs arise in the breaking: three of them can be identified with the SM GBs, a fourth one with the physical Higgs particle, while the remaining ten represent new scalar states. In ref. [6], it was shown that strong dynamical effects can induce large (i.e. $\mathcal{O}(f)$ ) masses for all the extra GBs, that therefore can be safely disregarded at 
low-energies, while still having a light (i.e. $\mathcal{O}(v)$ ) Higgs particle The discussion of this mechanism is beyond the scope of this letter. Moreover, in what follows, all the extra GBs are assumed heavy and removed from the low-energy spectrum, that contains only the four SM degrees of freedom.

For a comprehensive review of the notation used one can refer to ref. [40]. Here we only briefly recall the form of the $\boldsymbol{\Sigma}(x)$ field. We denote by $\mathcal{X}(x)$ the SU(5) embedding of the SM GBs:

$$
\mathcal{X}(x)=\frac{1}{\sqrt{2}}\left(\begin{array}{ccc}
0 & 0 & \mathbf{U}(x) e_{1} \\
0 & 0 & \mathbf{U}(x) e_{2} \\
\left(\mathbf{U}(x) e_{1}\right)^{\dagger} & \left(\mathbf{U}(x) e_{2}\right)^{\dagger} & 0
\end{array}\right)
$$

with $\mathbf{U}(x)$ defined as in eq. (1.1) and $e_{1}^{T}=(1,0), e_{2}^{T}=(0,1)$. Once the extra GBs are integrated out, at energies below $f$ the GB chiral field $\boldsymbol{\Sigma}(x)$ can be approximated by:

$$
\boldsymbol{\Sigma}(x) \approx e^{i \frac{\varphi(x)}{f} \mathcal{X}(x)} .
$$

with $\varphi(x)$ identified as the field driving the EW symmetry breaking and acquiring a nonvanishing vev,

$$
\frac{\varphi(x)}{f} \equiv \frac{h(x)+\langle\varphi\rangle}{f}=\left(\frac{h(x)+\langle\varphi\rangle}{v}\right) \sqrt{\xi}
$$

and $h(x)$ referring to the physical Higgs particle. Thanks to the special form of the $\mathcal{X}(x)$, one can easily obtain the following expression for $\boldsymbol{\Sigma}(x)$ :

$$
\boldsymbol{\Sigma}(x)=\mathbb{1}+i \sin \left(\frac{\varphi(x)}{f}\right) \mathcal{X}(x)+\left(\cos \left(\frac{\varphi(x)}{f}\right)-1\right) \mathcal{X}^{2}(x) .
$$

The last ingredient that needs to be specified is the embedding of the $\mathrm{SU}(2)_{L} \times \mathrm{U}(1)_{Y}$ generators in $\mathrm{SU}(5)$ :

$$
Q_{L}^{a}=\frac{1}{2}\left(\begin{array}{ccc}
\sigma_{a} & & \\
& \sigma_{a} & \\
& & 0
\end{array}\right), \quad Q_{Y}=\frac{1}{2}\left(\begin{array}{ccc}
-\mathbb{1}_{2} & & \\
& \mathbb{1}_{2} & \\
& & 0
\end{array}\right),
$$

where $\sigma_{a}$ denote the Pauli matrices and the normalisation of the generators has been chosen such that $\operatorname{Tr}\left(Q_{a} Q_{a}\right)=1$.

The global $\mathrm{SU}(2)_{L} \times \mathrm{SU}(2)_{R}$ symmetry can be embedded in the residual $\mathrm{SO}(5)$ group and this implies an approximate custodial symmetry conservation.

\subsubsection{The low-energy effective EW chiral Lagrangian}

Having the explicit expressions for $\boldsymbol{\Sigma}(x), \widetilde{\mathbf{V}}_{\mu}, \widetilde{\mathbf{W}}_{\mu}$ and $\widetilde{\mathbf{B}}_{\mu}$ and substituting them in the operators of the high-energy basis of eq. (3.18) one derives the low-energy effective Lagrangian, $\mathscr{L}_{\text {low, }, P}$ for the Georgi-Kaplan model as a function of the SM would-be GBs, the light scalar field $\varphi(x)$ and the SM gauge bosons. 
The low-energy projection of the four-derivative effective operators of eq. (3.18) gives:

$$
\begin{aligned}
\widetilde{\mathcal{B}}_{W W^{*}} & =\mathcal{S}_{W W^{*}} \\
\widetilde{\mathcal{B}}_{B \Sigma^{*}} & =-4 \mathcal{S}_{B B^{*}}+4 \sin ^{2}\left[\frac{\varphi}{2 f}\right] \mathcal{S}_{B B^{*}}, \\
\widetilde{\mathcal{B}}_{W \Sigma^{*}} & =-4 \mathcal{S}_{W W^{*}}+4 \sin ^{2}\left[\frac{\varphi}{2 f}\right] \mathcal{S}_{W W^{*}} \\
\widetilde{\mathcal{B}}_{1} & =\frac{1}{2} \sin ^{2}\left[\frac{\varphi}{2 f}\right] \mathcal{S}_{1} \\
\widetilde{\mathcal{B}}_{2} & =4 \sin ^{4}\left[\frac{\varphi}{2 f}\right]\left(\mathcal{S}_{B B^{*}}-\mathcal{S}_{W W^{*}}\right)+2 \sqrt{\xi} \cos \left[\frac{\varphi}{2 f}\right] \sin ^{3}\left[\frac{\varphi}{2 f}\right]\left(\mathcal{S}_{2}+2 \mathcal{S}_{3}\right), \\
\widetilde{\mathcal{B}}_{3} & =0
\end{aligned}
$$

The Higgs-independent part of the operator $\widetilde{\mathcal{B}}_{B \Sigma^{*}}$ can be safely neglected at lowenergy being equivalent to a total derivative and vanishing. On the other side, the Higgsindependent part of the operators $\widetilde{\mathcal{B}}_{W W^{*}}$ and $\widetilde{\mathcal{B}}_{W \Sigma^{*}}$ does not vanish as it provides CP-odd non-perturbative contributions. The last operator in the list, $\widetilde{\mathcal{B}}_{3}$ automatically vanishes in $\mathrm{SU}(5) / \mathrm{SO}(5)$ model due to the specific properties of the coset generators. Consequently at low-energy only four independent CP-odd perturbative couplings are relevant: the Higgsdependent parts contained in $\widetilde{\mathcal{B}}_{B \Sigma^{*}}, \widetilde{\mathcal{B}}_{W \Sigma^{*}}, \widetilde{\mathcal{B}}_{1}$ and $\widetilde{\mathcal{B}}_{2}$.

\subsection{The minimal $\mathrm{SO}(5) / \mathrm{SO}(4)$ composite Higgs model}

A very well know $\mathrm{CH}$ model is based on the coset $\mathrm{SO}(5) / \mathrm{SO}(4)$ [11], with only four GBs arising from the global symmetry breaking, that can be identified to the SM GBs and the Higgs field. Moreover as the preserved group $\mathrm{SO}(4)$ contains $\mathrm{SU}(2)_{L} \times \mathrm{SU}(2)_{R}$, custodial violating effects can arise only through SM-like sources, i.e. proportional to the hypercharge (or fermion couplings when introduced).

Due to the minimality of the GB content $\boldsymbol{\Sigma}(x)$ exactly reduces to

$$
\boldsymbol{\Sigma}(x)=e^{i \frac{\varphi(x)}{f} \mathcal{X}(x)} .
$$

with the GB non-linear field given by

$$
\mathcal{X}(x)=-\frac{i}{\sqrt{2}} \operatorname{Tr}\left(\mathbf{U} \sigma_{\hat{a}}\right) X_{\hat{a}}, \quad \hat{a}=1, \ldots, 4,
$$

where $\sigma_{\hat{a}} \equiv\left\{\sigma_{1}, \sigma_{2}, \sigma_{3}, i \mathbb{1}_{2}\right\}$ and the $\mathrm{SO}(5) / \mathrm{SO}(4)$ generators can be written in a compact form as:

$$
\left(X_{\hat{a}}\right)_{i j}=\frac{i}{\sqrt{2}}\left(\delta_{i 5} \delta_{j \hat{a}}-\delta_{j 5} \delta_{i \hat{a}}\right), \quad \hat{a}=1, \ldots, 4,
$$

Alike to the case of the Georgi-Kaplan model, the field $\boldsymbol{\Sigma}(x)$ takes the simple form in terms of linear and quadratic powers of $\mathcal{X}(x)$ shown in eq. (4.4). 
Finally, the embedding of the $\mathrm{SU}(2)_{L} \times \mathrm{U}(1)_{Y}$ generators in $\mathrm{SO}(5)$ reads:

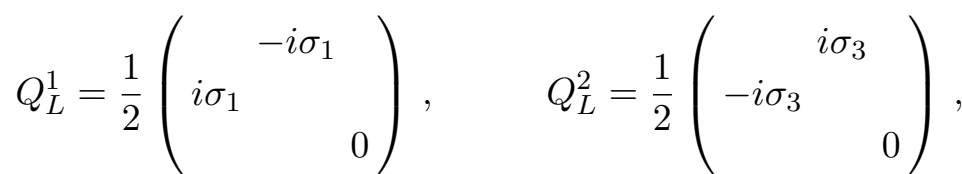

$$
\begin{aligned}
& Q_{L}^{3}=\frac{1}{2}\left(\begin{array}{ccc}
\sigma_{2} & & \\
& \sigma_{2} & \\
& & 0
\end{array}\right), \quad Q_{Y}=\frac{1}{2}\left(\begin{array}{ccc}
\sigma_{2} & & \\
& -\sigma_{2} & \\
& & 0
\end{array}\right) .
\end{aligned}
$$

\subsubsection{The low-energy effective EW chiral Lagrangian}

The $\mathscr{L}_{\text {low.CP }}$ Lagrangian originated from the projection of the basis in eq. (3.18) at low energies for the $\mathrm{SO}(5) / \mathrm{SO}(4)$ minimal $\mathrm{CH}$ setup turns out to be the same as that for the $\mathrm{SU}(5) / \mathrm{SO}(5)$ model. Indeed, on one side, the extra GBs present only in the $\mathrm{SU}(5) / \mathrm{SO}(5)$ model are heavy and therefore the spectrum content is the same for both models; on the other side, the gauging of only the SM group washes out the differences between the two preserved subgroups: a unitary transformation can be found linking the GB matrices of the two setups, showing that they are equivalent at low-energy. As already discussed in ref. [40] for the CP-even case, this suggests that the low-energy effective chiral Lagrangian for a minimal number of GBs, that can be arranged in a doublet of $\mathrm{SU}(2)_{L}$, and approximate custodial symmetry, is the same regardless of the specific ultraviolet completion.

\subsection{The $\mathrm{SU}(3) /(\mathrm{SU}(2) \times \mathrm{U}(1))$ composite Higgs model}

The last setup considered is the $\mathrm{SU}(3) /(\mathrm{SU}(2) \times \mathrm{U}(1)) \mathrm{CH}$ model. This setup is minimal, as it contains only four GBs, but contrary to the models previously considered, the custodial $\mathrm{SO}(4)$ is not contained in the preserved group $\mathcal{H}$, and therefore there is no (approximate) custodial symmetry. One should be aware that this leads to a large tree-level contribution to the $T$ parameter, that therefore requires some level of fine-tuning on the parameter $\xi$, making this setup somehow less attractive. Nevertheless, the study of the low-energy projection is instructive: although in the initial high-energy $\mathrm{SU}(3) /(\mathrm{SU}(2) \times \mathrm{U}(1))$ Lagrangian no extra sources of custodial breaking besides the SM (gauge) one are present, through the decomposition procedure, custodial breaking effects are generated in the low-energy effective Lagrangian.

Due to the minimality of the GB content the $\boldsymbol{\Sigma}(x)$ exactly reduces to

$$
\boldsymbol{\Sigma}(x)=e^{i \frac{\varphi(x)}{f} \mathcal{X}(x)},
$$

with the GB non-linear field given by

$$
\mathcal{X}(x)=\left(\begin{array}{cc}
0 & \mathbf{U}(x) e_{2} \\
\left(\mathbf{U}(x) e_{2}\right)^{\dagger} & 0
\end{array}\right) .
$$

As for the two models previously analysed, the GB field matrix $\boldsymbol{\Sigma}(x)$ can be expressed in terms of $\mathcal{X}(x)$ as in eq. (4.4). Finally the $\mathrm{SU}(3)$-embedding of the $\mathrm{SU}(2)_{L} \times \mathrm{U}(1)_{Y}$ 
generators are given by

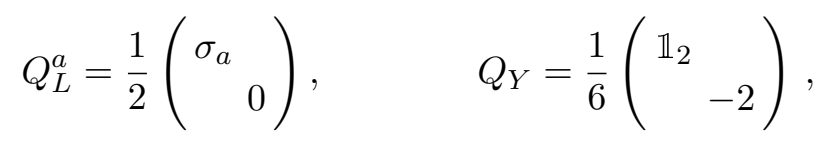

with $^{4} \operatorname{Tr}\left(Q_{L}^{a} Q_{L}^{a}\right)=1 / 2$ and $\operatorname{Tr}\left(Q_{Y} Q_{Y}\right)=1 / 6$.

\subsubsection{The low-energy effective EW chiral Lagrangian}

The low-energy Lagrangian $\mathscr{L}_{\text {low,ex }}$ is obtained by substituting the explicit expressions for $\boldsymbol{\Sigma}(x), \widetilde{\mathbf{V}}_{\mu}, \widetilde{\mathbf{W}}_{\mu}$ and $\widetilde{\mathbf{B}}_{\mu}$ in the operators of the high-energy basis in eq. (3.18). The lowenergy projection of the four-derivative operators listed in eq. (3.18) results in the following decomposition for the $\mathrm{SU}(3) /(\mathrm{SU}(2) \times \mathrm{U}(1))$ model: ${ }^{5}$

$$
\begin{aligned}
\widetilde{\mathcal{B}}_{W W^{*}} & =\frac{1}{2} \mathcal{S}_{W W^{*}}, \\
\widetilde{\mathcal{B}}_{B \Sigma^{*}} & =-\frac{1}{6}\left(1+3 \cos \left[\frac{2 \varphi}{f}\right]\right) \mathcal{S}_{B B^{*}}, \\
\widetilde{\mathcal{B}}_{W \Sigma^{*}} & =-2 \cos \left[\frac{\varphi}{f}\right] \mathcal{S}_{W W^{*}}+\frac{1}{2} \sin ^{4}\left[\frac{\varphi}{2 f}\right] \mathcal{S}_{8}, \\
\widetilde{\mathcal{B}}_{1} & =\frac{1}{8} \sin ^{2}\left[\frac{\varphi}{f}\right] \mathcal{S}_{1}, \\
\widetilde{\mathcal{B}}_{2} & =\frac{1}{4} \sin ^{4}\left[\frac{\varphi}{f}\right]\left(\mathcal{S}_{B B^{*}}-\mathcal{S}_{W W^{*}}\right)+\frac{1}{4} \sqrt{\xi} \cos \left[\frac{\varphi}{f}\right] \sin ^{3}\left[\frac{\varphi}{f}\right]\left(\mathcal{S}_{2}+2 \mathcal{S}_{3}\right), \\
\widetilde{\mathcal{B}}_{3} & =0 .
\end{aligned}
$$

Notice, in particular, the presence of the custodial violating operator $\mathcal{S}_{8}$ in the decomposition of the $\widetilde{\mathcal{B}}_{W \Sigma^{*}}$ operator, that corresponds to a tree-level source of custodial symmetry breaking. Notice however that it does not contribute to the $T$ parameter and therefore no constraint can be put on its coefficient.

\section{Matching the high- and the low-energy Lagrangians}

The functions, appearing in the low-energy basis in each of the $\mathrm{CH}$ models considered, encode the dependence on the $h$ field: they turn out to be trigonometric due to the GB nature of the Higgs field in these setups. In the general $\mathscr{L}_{\text {low,EP }}$ basis, this dependence is encoded into the generic functions $\mathcal{F}_{i}(h)$ in eq. (2.10) and into some operators which contain derivatives of $h$. It is then possible to identify the products $c_{i} \mathcal{F}_{i}(h)$ in terms of the high-energy parameters, by comparing the low-energy EW chiral Lagrangian of the specific

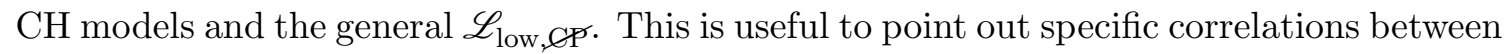
couplings that could help investigating the nature of the EWSB mechanism $[29,38,39]$.

Table 1 reports the expression of the products $c_{i} \mathcal{F}_{i}(h)$ for the three distinct $\mathrm{CH}$ setups considered before, only for the operators of the low-energy basis that indeed receive contributions.

\footnotetext{
${ }^{4} \mathrm{~A}$ typo is present in ref. [40] after eq. (6.6): $\operatorname{Tr}\left(Q_{L}^{a} Q_{L}^{a}\right)=1 / 2$ has been adopted also there.

${ }^{5}$ This projection is consistent with the one in ref. [40] in eq. (6.8), where a typo is present on the first two operators: the correct values are $\widetilde{\mathcal{A}}_{B}=\mathcal{P}_{B} / 6$, and $\widetilde{\mathcal{A}}_{W}=\mathcal{P}_{W} / 2$.
} 


\begin{tabular}{|c|cc|}
\hline$c_{i} \mathcal{F}_{i}(h)$ & $\mathrm{SU}(5) / \mathrm{SO}(5)$ & $\mathrm{SU}(3) /(\mathrm{SU}(2) \times \mathrm{U}(1))$ \\
\hline $\mathcal{F}_{B B^{*}}(h)$ & $\mathrm{SO}(5) / \mathrm{SO}(4)$ & $4 \tilde{c}_{B \Sigma^{*}} \sin ^{2} \frac{\varphi}{2 f} \cos ^{2} \frac{\varphi}{2 f}+4 \tilde{c}_{2} \sin ^{4} \frac{\varphi}{2 f} \cos ^{4} \frac{\varphi}{2 f}$ \\
$\mathcal{F}_{W W^{*}}(h)$ & $\sin ^{2} \frac{\varphi}{2 f}+4 \tilde{c}_{2} \sin ^{4} \frac{\varphi}{2 f}$ & $\frac{\tilde{c}_{1}}{2} \sin ^{2} \frac{\varphi}{2 f} \cos ^{2} \frac{\varphi}{2 f}$ \\
$c_{1} \mathcal{F}_{1}(h)$ & $\frac{\tilde{c}_{W W *}-4 \tilde{c}_{W \Sigma^{*}} \cos ^{2} \frac{\varphi}{2 f}-4 \tilde{c}_{2} \sin ^{4} \frac{\varphi}{2 f}}{\frac{\tilde{c}_{W}}{2} \sin ^{2} \frac{\varphi}{2 f}}-2 \tilde{c}_{W \Sigma^{*}}\left(1+2 \cos ^{2} \frac{\varphi}{2 f}\right)-4 \tilde{c}_{2} \sin ^{4} \frac{\varphi}{2 f} \cos ^{4} \frac{\varphi}{2 f}$ \\
$c_{2} \mathcal{F}_{2}(h)$ & $2 \tilde{c}_{2} \sqrt{\xi} \cos \frac{\varphi}{2 f} \sin ^{3} \frac{\varphi}{2 f}$ & $2 \tilde{c}_{2} \sqrt{\xi} \cos ^{3} \frac{\varphi}{2 f} \sin ^{3} \frac{\varphi}{2 f}\left(2 \cos ^{2} \frac{\varphi}{2 f}-1\right)$ \\
$c_{3} \mathcal{F}_{3}(h)$ & $4 \tilde{c}_{2} \sqrt{\xi} \cos \frac{\varphi}{2 f} \sin ^{3} \frac{\varphi}{2 f}$ & $4 \tilde{c}_{2} \sqrt{\xi} \cos ^{3} \frac{\varphi}{2 f} \sin ^{3} \frac{\varphi}{2 f}\left(2 \cos ^{2} \frac{\varphi}{2 f}-1\right)$ \\
\hline$c_{8} \mathcal{F}_{8}(h)$ & - & $\frac{\tilde{c}_{W \Sigma^{*}}}{2} \sin ^{4} \frac{\varphi}{2 f}$ \\
\hline
\end{tabular}

Table 1. Expressions for the products $c_{i} \mathcal{F}_{i}(h)$ for $\mathrm{SU}(5) / \mathrm{SO}(5)(\mathrm{SO}(5) / \mathrm{SO}(4))$ and $\mathrm{SU}(3) /(\mathrm{SU}(2) \times$ $\mathrm{U}(1)$ respectively. The "-" entry indicates no leading order contributions at low-energy to the corresponding operator.

Some interesting comments can be inferred from table 1 .

$\mathrm{SU}(5) / \mathrm{SO}(5)$ and $\mathrm{SO}(5) / \mathrm{SO}(4)$. All the custodial preserving operators entering the low-energy Lagrangian $\mathscr{L}_{\text {low,® }}$ result from the projection. As expected, no tree-level custodial breaking operator arises from the low-energy projection of $\mathrm{SU}(5) / \mathrm{SO}(5)$ and $\mathrm{SO}(5) / \mathrm{SO}(4)$ models, as they are naturally custodial preserving. Moreover, notice that $c_{2} \mathcal{F}_{2}(h)$ and $c_{3} \mathcal{F}_{3}(h)$ are functions of the same high-energy parameter $\tilde{c}_{2}$, implying a correlation between the couplings described by $\mathcal{S}_{2}$ and $\mathcal{S}_{3}$.

$\mathbf{S U}(\mathbf{3}) /(\mathbf{S U}(\mathbf{2}) \times \mathbf{U}(\mathbf{1}))$. Besides the custodial preserving operators, only one custodial breaking operator of the low-energy basis, $\mathcal{S}_{8}$, receives contributions from the projection. As for the previous $\mathrm{CH}$ models, the interactions described by $\mathcal{S}_{2}$ and $\mathcal{S}_{3}$ turn out to be correlated.

Furthermore, notice that the arbitrary functions $\mathcal{F}_{i}(h)$ of the generic low-energy effective chiral Lagrangian become now a constrained set, as a consequence of having chosen a specific $\mathrm{CH}$ model.

\subsection{The small $\xi$ limit}

It is particularly interesting to consider the $f \gg v$, or equivalently $\xi \ll 1$, limit. In fact in this limit the non-linear $\mathrm{CH}$ model should overlap with the case in which the EWSB is linearly realised and the Lagrangian written in terms of the Higgs as an $\mathrm{SU}(2)_{L}$ doublet. For example, taking the operators in eq. (4.6) for the $\mathrm{SU}(5) / \mathrm{SO}(5)$ setup and expanding them in Taylor series in $1 / f$ as defined in eq. (4.3), one concludes that at first order in $\xi$,

$$
\begin{aligned}
\widetilde{\mathcal{B}}_{B \Sigma^{*}} & \approx \xi(1+h / v)^{2} \mathcal{S}_{B B^{*}}-4 \mathcal{S}_{B B^{*}} \\
\widetilde{\mathcal{B}}_{W \Sigma^{*}} & \approx \xi(1+h / v)^{2} \mathcal{S}_{W W^{*}}-4 \mathcal{S}_{W W^{*}} \\
\widetilde{\mathcal{B}}_{1} & \approx \frac{1}{8} \xi(1+h / v)^{2} \mathcal{S}_{1} \\
\widetilde{\mathcal{B}}_{2} & \approx 0
\end{aligned}
$$




\begin{tabular}{|c|c|c|}
\hline$c_{i} \mathcal{F}_{i}(h)$ & $\begin{array}{l}\mathrm{SU}(5) / \mathrm{SO}(5) \\
\mathrm{SO}(5) / \mathrm{SO}(4)\end{array}$ & $\mathrm{SU}(3) /(\mathrm{SU}(2) \times \mathrm{U}(1))$ \\
\hline $\mathcal{F}_{B B^{*}}(h)$ & $\tilde{c}_{B \Sigma^{*}} \xi\left(1+\frac{h}{v}\right)^{2}+\mathcal{O}\left(\xi^{2}\right)$ & $\tilde{c}_{B \Sigma^{*}} \xi\left(1+\frac{h}{v}\right)^{2}+\mathcal{O}\left(\xi^{2}\right)$ \\
\hline $\begin{array}{l}\mathcal{F}_{W W^{*}}(h) \\
c_{1} \mathcal{F}_{1}(h)\end{array}$ & $\begin{array}{c}\tilde{c}_{W W *}+\tilde{c}_{W \Sigma^{*}}\left(-4+\xi\left(1+\frac{h}{v}\right)^{2}\right)+\mathcal{O}\left(\xi^{2}\right) \\
\frac{\tilde{c}_{1}}{8} \xi\left(1+\frac{h}{v}\right)^{2}+\mathcal{O}\left(\xi^{2}\right)\end{array}$ & $\begin{array}{c}\frac{\tilde{c}_{W W *}}{2}+\tilde{c}_{W \Sigma^{*}}\left(-6+\xi\left(1+\frac{h}{v}\right)^{2}\right)+\mathcal{O}\left(\xi^{2}\right) \\
\frac{\tilde{c}_{1}}{8} \xi\left(1+\frac{h}{v}\right)^{2}+\mathcal{O}\left(\xi^{2}\right)\end{array}$ \\
\hline$c_{2} \mathcal{F}_{2}(h)$ & $\mathcal{O}\left(\xi^{2}\right)$ & $\mathcal{O}\left(\xi^{2}\right)$ \\
\hline$c_{3} \mathcal{F}_{3}(h)$ & $\mathcal{O}\left(\xi^{2}\right)$ & $\mathcal{O}\left(\xi^{2}\right)$ \\
\hline$c_{8} \mathcal{F}_{8}(h)$ & - & $\mathcal{O}\left(\xi^{2}\right)$ \\
\hline
\end{tabular}

Table 2. Expressions for the products $c_{i} \mathcal{F}_{i}(h)$ for $\mathrm{SU}(5) / \mathrm{SO}(5)(\mathrm{SO}(5) / \mathrm{SO}(4))$ and $\mathrm{SU}(3) /(\mathrm{SU}(2) \times$ $\mathrm{U}(1))$ in the $\xi \ll 1$ limit.

with $\widetilde{\mathcal{B}}_{2}$ giving contribution only at order $O\left(\xi^{2}\right)$. This procedure can be repeated with all the terms appearing in table 1 and the results can be read in table 2 . This should be compared with the effective $d=6 \mathrm{CP}$-odd Lagrangian in the linear regime [44, 45]: only three operators form the EW bosonic basis:

$$
\begin{aligned}
\mathcal{Q}_{\varphi \widetilde{B}} & =\mathbf{B}_{\mu \nu}^{*} \mathbf{B}^{\mu \nu} \Phi^{\dagger} \Phi \\
\mathcal{Q}_{\varphi \widetilde{W}} & =\Phi^{\dagger} \mathbf{W}_{\mu \nu}^{*} \mathbf{W}^{\mu \nu} \Phi \\
\mathcal{Q}_{\varphi \widetilde{B} W} & =\mathbf{B}_{\mu \nu}^{*} \Phi^{\dagger} \mathbf{W}^{\mu \nu} \Phi
\end{aligned}
$$

and own physical interactions with perturbative effects. There is one-to-one correspondence between these two classes of operators: $\mathcal{Q}_{\varphi \widetilde{B}} \leftrightarrow \widetilde{\mathcal{B}}_{B \Sigma^{*}}, \mathcal{Q}_{\varphi \widetilde{W}} \leftrightarrow \widetilde{\mathcal{B}}_{W \Sigma^{*}}$ and $\mathcal{Q}_{\varphi \widetilde{B} W} \leftrightarrow \widetilde{\mathcal{B}}_{1}$. Conversely, $\widetilde{\mathcal{B}}_{2}$ contributes at low-energy to $\mathcal{S}_{2}$ and $\mathcal{S}_{3}$, but only at $\xi^{2}$, i.e. its linear sibling ${ }^{6}$ should have $d=8$ : indeed, by using integration by parts and the Bianchi identities, it is straightforward to verify that the interactions of $\mathcal{S}_{2}$ and $\mathcal{S}_{3}$ are described at the lowest order in the linear expansion by the operators

$$
\mathbf{B}_{\mu \nu}^{*}\left(\Phi^{\dagger} \stackrel{\leftrightarrow}{\mathbf{D}^{\mu}} \Phi\right) \mathbf{D}^{\nu}\left(\Phi^{\dagger} \Phi\right), \quad\left(\Phi^{\dagger} \stackrel{\leftrightarrow}{\mathbf{D}^{\mu}} \mathbf{W}_{\mu \nu}^{*} \Phi\right) \mathbf{D}^{\nu}\left(\Phi^{\dagger} \Phi\right)
$$

with $\mathbf{D}_{\mu} \Phi \equiv\left(\partial_{\mu}+\frac{i}{2} g^{\prime} B_{\mu}+\frac{i}{2} g \sigma_{i} W_{\mu}^{i}\right) \Phi$ and $\Phi^{\dagger} \stackrel{\leftrightarrow}{\mathbf{D}^{\mu}} \Phi \equiv \Phi^{\dagger} \mathbf{D}_{\mu} \Phi-\mathbf{D}_{\mu} \Phi^{\dagger} \Phi$

The products $c_{i} \mathcal{F}_{i}(h)$ corresponding to custodial-breaking operators are suppressed by $\xi^{2}$ and therefore they are also described in the linear expansion by $d=8$ operators. However, a complete comparison is not possible in this case, as no $d=8$ basis has been defined yet, to our knowledge.

\footnotetext{
${ }^{6}$ This is in contrast with eq. (A.1) in ref. [29], where two $d=6$ linear operators have been indicated as siblings of $\mathcal{S}_{2}$ and $\mathcal{S}_{3}$. Those operators do contain the interactions of $\mathcal{S}_{2}$ and $\mathcal{S}_{3}$, but they are not the lowest dimensional ones.
} 


\section{Conclusions}

The CP-odd Lagrangian for a generic $\mathcal{G} / \mathcal{H}$ symmetric coset has been constructed, focusing on the bosonic sector: only four operators constitute a basis. Once considering the gauging of the hypercharge as the only source of custodial symmetry breaking, the number of operators increases up to six, including the topological term. These operators are written in terms of the SM gauge bosons, embedded in $\mathcal{G}$ representations, and of the matrix for the Goldstone Bosons, which in these contexts also includes the physical Higgs fields. Once a specific coset is considered, however, some of these operators could vanish or be redundant.

The projection of this Lagrangian at low-energy for three different $\mathrm{CH}$ models has allowed to confirm the subset of custodial preserving couplings of the general low-energy CP-odd Lagrangian for a dynamical Higgs developed in ref. [29] and leads to the identification of correlations among the effective low-energy operators and allows a comparison with the linear effective Lagrangian for an elementary Higgs. The results presented here are consistent with ref. [40], where only the CP-even sector has been considered. In particular, the low-energy projection of the $\mathrm{SU}(5) / \mathrm{SO}(5)$ and $\mathrm{SO}(5) / \mathrm{SO}(4)$ models turns out to be the same, due to the fact that neglecting the effects of the extra GBs of the first model and gauging only the SM group force the preserved subgroups in the two models to be isomorphic. On the other hand, some differences appear with the low-energy projection of $\mathrm{SU}(3) /(\mathrm{SU}(2) \times \mathrm{U}(1))$, that is not intrinsically custodial preserving.

The results also confirm the powers of $\xi$ predicted in ref. [29] as weights for each operator of the low-energy effective chiral Lagrangian, allowing an immediate comparison with linear expansions for an elementary Higgs. One can point out that the differences stem from the $h$ dependence: functions of $\sin [(\langle\varphi\rangle+h) / 2 f]$ for the $\mathrm{CH}$ models and powers of $(v+h) / 2$ for the linear realisation. When $\xi \ll 1$, the trigonometric dependence on $h$ reduces exactly to the linear one, as $\sin ^{2}(\varphi / f)=\xi(1+h / v)^{2}+\mathcal{O}\left(\xi^{2}\right)$, neglecting the higher order terms in $\xi$. This result suggests that the use of the linear expansion to construct $\mathrm{CH}$ model Lagrangians can be justified in this limit. On the other hand, if $\xi$ is not so small, the deviations from the linear structure $(1+h / v)$ could be significant and therefore comparing observables with different Higgs legs could disentangle an elementary from a composite Higgs scenario.

Finally, to distinguish between the doublet or non-doublet nature of the Higgs, it is then necessary to compare pure-gauge and gauge-Higgs couplings [29, 38, 39], whose precise form we have determined here for the specific $\mathrm{CH}$ models considered. The strength of these observables depends on $\xi$ and therefore the larger $\xi$ the easier it will be to detect a signal that may be able to shed light on the Higgs representation.

\section{Acknowledgments}

We thank Ilaria Brivio, Roberto Contino, Belen Gavela, Elizabeth Jenkins, Aneesh Manohar and Andrea Wulzer for constructive conversations. The work of I.M.H. is supported by an ESR contract of the European Union network FP7 ITN INVISIBLES (Marie Curie Actions, PITN-GA-2011-289442). L.M. acknowledges partial support of the Eu- 
ropean Union network FP7 ITN INVISIBLES, of CiCYT through the project FPA201231880, of the Spanish MINECO's "Centro de Excelencia Severo Ochoa" Programme under grant SEV-2012-0249, and by a grant from the Simons Foundation. S.R. acknowledges partial support of the European Union network FP7 ITN INVISIBLES and of the COFIN program PRIN 2010. This work was partially performed at the Aspen Center for Physics, which is supported by National Science Foundation grant PHY-1066293.

Open Access. This article is distributed under the terms of the Creative Commons Attribution License (CC-BY 4.0), which permits any use, distribution and reproduction in any medium, provided the original author(s) and source are credited.

\section{References}

[1] D.B. Kaplan and H. Georgi, SU(2) $\times$ U(1) Breaking by Vacuum Misalignment, Phys. Lett. B 136 (1984) 183 [INSPIRE].

[2] D.B. Kaplan, H. Georgi and S. Dimopoulos, Composite Higgs Scalars, Phys. Lett. B 136 (1984) 187 [INSPIRE].

[3] T. Banks, Constraints on $\mathrm{SU}(2) \times \mathrm{U}(1)$ Breaking by Vacuum Misalignment, Nucl. Phys. B 243 (1984) 125 [INSPIRE].

[4] H. Georgi, D.B. Kaplan and P. Galison, Calculation of the Composite Higgs Mass, Phys. Lett. B 143 (1984) 152 [inSPIRE].

[5] H. Georgi and D.B. Kaplan, Composite Higgs and Custodial SU(2), Phys. Lett. B 145 (1984) 216 [INSPIRE].

[6] M.J. Dugan, H. Georgi and D.B. Kaplan, Anatomy of a Composite Higgs Model, Nucl. Phys. B 254 (1985) 299 [INSPIRE].

[7] A. Manohar and H. Georgi, Chiral Quarks and the Nonrelativistic Quark Model, Nucl. Phys. B 234 (1984) 189 [INSPIRE].

[8] R. Contino, The Higgs as a Composite Nambu-Goldstone Boson, arXiv:1005.4269 [INSPIRE].

[9] G. Panico and A. Wulzer, The Composite Nambu-Goldstone Higgs, Lect. Notes Phys. 913 (2016) pp.1-316 [arXiv: 1506.01961] [InSPIRE].

[10] R. Contino, Y. Nomura and A. Pomarol, Higgs as a holographic pseudoGoldstone boson, Nucl. Phys. B 671 (2003) 148 [hep-ph/0306259] [INSPIRE].

[11] K. Agashe, R. Contino and A. Pomarol, The minimal composite Higgs model, Nucl. Phys. B 719 (2005) 165 [hep-ph/0412089] [INSPIRE].

[12] R. Contino, L. Da Rold and A. Pomarol, Light custodians in natural composite Higgs models, Phys. Rev. D 75 (2007) 055014 [hep-ph/0612048] [INSPIRE].

[13] B. Gripaios, A. Pomarol, F. Riva and J. Serra, Beyond the Minimal Composite Higgs Model, JHEP 04 (2009) 070 [arXiv: 0902.1483] [InSPIRE].

[14] O. Matsedonskyi, G. Panico and A. Wulzer, Light Top Partners for a Light Composite Higgs, JHEP 01 (2013) 164 [arXiv:1204.6333] [InSPIRE]. 
[15] A. Pomarol and F. Riva, The Composite Higgs and Light Resonance Connection, JHEP 08 (2012) 135 [arXiv: 1205.6434] [INSPIRE].

[16] M. Redi and A. Tesi, Implications of a Light Higgs in Composite Models, JHEP 10 (2012) 166 [arXiv: 1205.0232] [INSPIRE].

[17] D. Marzocca, M. Serone and J. Shu, General Composite Higgs Models, JHEP 08 (2012) 013 [arXiv: 1205.0770] [INSPIRE].

[18] G. Panico, M. Redi, A. Tesi and A. Wulzer, On the Tuning and the Mass of the Composite Higgs, JHEP 03 (2013) 051 [arXiv: 1210.7114] [INSPIRE].

[19] D. Pappadopulo, A. Thamm and R. Torre, A minimally tuned composite Higgs model from an extra dimension, JHEP 07 (2013) 058 [arXiv: 1303.3062] [INSPIRE].

[20] R. Contino, C. Grojean, D. Pappadopulo, R. Rattazzi and A. Thamm, Strong Higgs Interactions at a Linear Collider, JHEP 02 (2014) 006 [arXiv: 1309.7038] [INSPIRE].

[21] O. Matsedonskyi, F. Riva and T. Vantalon, Composite Charge 8/3 Resonances at the LHC, JHEP 04 (2014) 059 [arXiv:1401.3740] [INSPIRE].

[22] ATLAS collaboration, Observation of a new particle in the search for the Standard Model Higgs boson with the ATLAS detector at the LHC, Phys. Lett. B 716 (2012) 1 [arXiv: 1207.7214] [INSPIRE].

[23] CMS collaboration, Observation of a new boson at a mass of $125 \mathrm{GeV}$ with the CMS experiment at the LHC, Phys. Lett. B 716 (2012) 30 [arXiv:1207.7235] [INSPIRE].

[24] R. Alonso, M.B. Gavela, L. Merlo, S. Rigolin and J. Yepes, The Effective Chiral Lagrangian for a Light Dynamical "Higgs Particle", Phys. Lett. B 722 (2013) 330 [Erratum ibid. B 726 (2013) 926] [arXiv: 1212.3305] [INSPIRE].

[25] R. Alonso, M.B. Gavela, L. Merlo, S. Rigolin and J. Yepes, Minimal Flavour Violation with Strong Higgs Dynamics, JHEP 06 (2012) 076 [arXiv: 1201.1511] [INSPIRE].

[26] R. Alonso, M.B. Gavela, L. Merlo, S. Rigolin and J. Yepes, Flavor with a light dynamical "Higgs particle", Phys. Rev. D 87 (2013) 055019 [arXiv:1212.3307] [INSPIRE].

[27] G. Buchalla and O. Catà, Effective Theory of a Dynamically Broken Electroweak Standard Model at NLO, JHEP 07 (2012) 101 [arXiv: 1203.6510] [INSPIRE].

[28] G. Buchalla, O. Cata and C. Krause, Complete Electroweak Chiral Lagrangian with a Light Higgs at NLO, Nucl. Phys. B 880 (2014) 552 [arXiv:1307.5017] [InSPIRE].

[29] M.B. Gavela, J. Gonzalez-Fraile, M.C. Gonzalez-Garcia, L. Merlo, S. Rigolin and J. Yepes, CP violation with a dynamical Higgs, JHEP 10 (2014) 44 [arXiv:1406.6367] [INSPIRE].

[30] T. Appelquist and C.W. Bernard, Strongly Interacting Higgs Bosons, Phys. Rev. D 22 (1980) 200 [INSPIRE].

[31] A.C. Longhitano, Heavy Higgs Bosons in the Weinberg-Salam Model, Phys. Rev. D 22 (1980) 1166 [INSPIRE].

[32] A.C. Longhitano, Low-Energy Impact of a Heavy Higgs Boson Sector, Nucl. Phys. B 188 (1981) 118 [INSPIRE].

[33] F. Feruglio, The chiral approach to the electroweak interactions, Int. J. Mod. Phys. A 8 (1993) 4937 [hep-ph/9301281] [INSPIRE]. 
[34] T. Appelquist and G.-H. Wu, The electroweak chiral Lagrangian and new precision measurements, Phys. Rev. D 48 (1993) 3235 [hep-ph/9304240] [InSPIRE].

[35] B. Grinstein and M. Trott, A Higgs-Higgs bound state due to new physics at a TeV, Phys. Rev. D 76 (2007) 073002 [arXiv: 0704.1505] [INSPIRE].

[36] R. Contino, C. Grojean, M. Moretti, F. Piccinini and R. Rattazzi, Strong Double Higgs Production at the LHC, JHEP 05 (2010) 089 [arXiv: 1002.1011] [INSPIRE].

[37] A. Azatov, R. Contino and J. Galloway, Model-Independent Bounds on a Light Higgs, JHEP 04 (2012) 127 [Erratum ibid. 1304 (2013) 140] [arXiv: 1202.3415] [INSPIRE].

[38] I. Brivio et al., Disentangling a dynamical Higgs, JHEP 03 (2014) 024 [arXiv:1311.1823] [INSPIRE].

[39] I. Brivio, O.J.P. Éboli, M.B. Gavela, M.C. Gonzalez-Garcia, L. Merlo and S. Rigolin, Higgs ultraviolet softening, JHEP 12 (2014) 004 [arXiv:1405.5412] [INSPIRE].

[40] R. Alonso, I. Brivio, B. Gavela, L. Merlo and S. Rigolin, Sigma Decomposition, JHEP 12 (2014) 034 [arXiv: 1409.1589] [INSPIRE].

[41] I. Low, Adler's zero and effective Lagrangians for nonlinearly realized symmetry, Phys. Rev. D 91 (2015) 105017 [arXiv:1412.2145] [INSPIRE].

[42] S.R. Coleman, J. Wess and B. Zumino, Structure of phenomenological Lagrangians. 1., Phys. Rev. 177 (1969) 2239 [inSPIRE].

[43] C.G. Callan Jr., S.R. Coleman, J. Wess and B. Zumino, Structure of phenomenological Lagrangians. 2., Phys. Rev. 177 (1969) 2247 [INSPIRE].

[44] W. Buchmüller and D. Wyler, Effective Lagrangian Analysis of New Interactions and Flavor Conservation, Nucl. Phys. B 268 (1986) 621 [INSPIRE].

[45] B. Grzadkowski, M. Iskrzynski, M. Misiak and J. Rosiek, Dimension-Six Terms in the Standard Model Lagrangian, JHEP 10 (2010) 085 [arXiv: 1008.4884] [INSPIRE]. 